\title{
ADULTOS HIPERTENSOS HOSPITALIZADOS: PERCEPÇÃO DE GRAVIDADE DA DOENÇA E DE QUALIDADE DE VIDA
}

\author{
Maria Gorette dos Reis ${ }^{1}$ \\ Regiane de Quadros Glashan ${ }^{2}$
}

Reis MG dos, Glashan RQ. Adultos hipertensos hospitalizados: percepção de gravidade da doença e de qualidade de vida. Rev Latino-am Enfermagem 2001 maio; 9(3):51-7.

Estudo descritivo que objetivou identificar a percepção de qualidade de vida em hipertensos adultos hospitalizados, confrontando os dados relacionados à qualidade de vida com a percepção de gravidade da doença. A população abrangeu 83 pacientes internados na Clínica Médica do Núcleo de Hospital Universitário da UFMS. Os dados foram coletados por meio de entrevista utilizando-se instrumento genérico de avaliação de saúde traduzido e validado no Brasil, o SF-36®. Os principais resultados mostraram que, sob a ótica dos pacientes estudados, a percepção de qualidade de vida é boa, assim como a percepção de ausência de severidade da doença.

UNITERMOS: qualidade de vida, enfermagem

\section{HOSPITALIZED HYPERTENSIVE ADULTS: PERCEPTIONS OF DISEASE GRAVITY AND OF LIFE QUALITY}

The purpose of this descriptive study was to identify the perception of life quality in hospitalized hypertensive adults, confronting the data related to life quality with perceptions of disease gravity. The population comprised 83 patients hospitalized in the Medical Clinic of the University Hospital at the Federal University of Mato Grosso do Sul (UFMS). The data were collected by means of interviews using a health evaluation instrument that had been translated and validated in Brazil, the SF-36®. The main results showed that, in view of the studied patients, the perception of life quality as well as the perception of absence of disease gravity were good.

KEY WORDS: quality of life, nursing

\section{ADULTOS HIPERTENSOS HOSPITALIZADOS: PERCEPCIÓN DE LA GRAVEDAD DE LA ENFERMEDAD Y DE LA CALIDAD DE VIDA}

Estudio descriptivo que tuvo como objetivo identificar la percepción de calidad de vida en adultos hipertensos hospitalizados, confrontando los datos relacionados con la calidad de vida con la percepción de gravedad de la enfermedad. La población constó de 83 pacientes internados en la Clínica Médica del Núcleo de Hospital Universitario de la UFMS. Los datos fueron recolectados por medio de entrevista usando un instrumento genérico de evaluación de salud traducido y validado en el Brazil, el SF-36®. Los resultados principales mostraron que, bajo la óptica de los pacientes estudiados, la percepción de calidad de vida es buena, así como la percepción de ausencia de severidad de la enfermedad.

TÉRMINOS CLAVES: calidad de vida, enfermería

\footnotetext{
${ }^{1}$ Enfermeira, Professor do Departamento de Enfermagem da Universidade Federal de Mato Grosso do Sul - UFMS. Mestre pela Universidade Federal de São Paulo - Escola Paulista de Medicina-UNIFESP-EPM - Área Saúde do Adulto. E-mail: mgorette@nin.ufms.br

${ }^{2}$ Enfermeira, Professor Doutor do Departamento de Enfermagem - Área Médico-Cirúrgica da Universidade Federal de São Paulo - Escola Paulista de Medicina-UNIFESP-EPM
} 


\section{INTRODUÇÃO}

As atuais condições de vida, trabalho, modificações econômicas e políticas, assim como a expectativa de vida aumentada, tem gerado uma elevação significativa de doenças crônicodegenerativas, especialmente as doenças do aparelho circulatório, dentre elas a hipertensão arterial ${ }^{(1-2)}$.

A hipertensão arterial (HA) é descrita por vários autores como uma doença crônica, multifatorial, de detecção quase sempre tardia, devido ao seu curso assintomático e prolongado, que apresenta elevada prevalência, sendo considerada como o principal fator de risco de morbi-mortalidade cardiovascular ${ }^{(3-6)}$.

Segundo o III Consenso Brasileiro de Hipertensão Arterial, "estima-se que $15 \%$ a $20 \%$ da população brasileira pode ser rotulada como hipertensa" ${ }^{(7)}$. Na América Latina há mais de 30 milhões de hipertensos, com pequenas variações entre os países.

Freqüentemente, a HA resulta em graves complicações como insuficiência cardíaca, renal e acidente vascular cerebral, podendo portanto evoluir para danos severos, com hospitalização de pacientes. Devido a sua cronicidade, causa agravos sociais por absenteísmo ao trabalho, elevados custos com internações de longa permanência, incapacitação por invalidez, aposentadoria precoce e outros $^{(8)}$.

Além disso, constitui-se num desafio para a equipe de saúde em propor uma terapêutica eficaz a longo prazo, porque requer a participação e cooperação do portador, o qual convive com a cronicidade e tem dificuldade em alterar seu estilo de vida ${ }^{(9)}$.

A Organização Mundial de Saúde (OMS) define como hipertenso o indivíduo adulto, maior de 18 anos, com valores de pressão arterial sistólica (PAS) igual ou maior que $160 \mathrm{mmHg}$ ou pressão arterial diastólica (PAD) igual ou maior que $95 \mathrm{mmHg}$. Os valores intermediários são considerados limítrofes ${ }^{(10)}$.

A hipertensão primária ou essencial, de origem desconhecida, representa em torno de $90 \%$ dos casos de hipertensão. A hipertensão secundária, relacionada a um processo patológico, representa $5 \%$ a $10 \%$ dos $\operatorname{casos}^{(11)}$.

Segundo o Ministério da Saúde, fatores de risco constitucionais (idade, sexo, fatores genéticos como raça, história familiar e obesidade) e fatores ambientais (ingestão de sal, álcool, drogas, gorduras, tabagismo, estresse e sedentarismo), contribuem para o desenvolvimento da doença ${ }^{(10)}$. A HA por si mesma já se constitui um importante fator de risco, principalmente para doenças cerebrovasculares e cardiovasculares, como por exemplo o infarto agudo do miocárdio ${ }^{(12)}$.

Desta forma, relaciona-se a constatação freqüente entre hipertensos: um estilo de vida que propicia desenvolvimento e/ou manutenção de fatores que comprovadamente contribuem para a ocorrência de complicações.
A terapêutica, incluindo as modificações no estilo de vida relacionadas ao ambiente, hábitos pessoais e organização da atenção à saúde, além de proporcionar alívio dos sintomas, traduzse em melhoria da qualidade de vida (QV) do hipertenso.

A QV é definida como a "sensação de bem-estar físico e de satisfação emocional, psíquica e social do indivíduo"(13)

A severidade, a duração da doença e a resposta individual à terapêutica podem influenciar a QV. No entanto, a percepção que o indivíduo tem do seu estado de saúde também pode interferir na sua QV, inclusive porque das suas crenças e dos seus valores depende em grande parte a sua satisfação de viver ${ }^{(14)}$.

Portanto, se a forma como o indivíduo percebe sua enfermidade pode influenciar os mecanismos individuais para satisfazer suas necessidades e a busca dos serviços assistenciais ${ }^{(6)}$, a adesão ao tratamento e conseqüentemente a eficácia da assistência prestada, podem depender muito da percepção individual que ele tem a respeito da doença.

Na literatura encontra-se melhor compreensão quanto a dimensão da gravidade da doença percebida: "a gravidade da doença pode ser avaliada tanto pelo grau de perturbação emocional criada ao pensar na doença, quanto pelos tipos de conseqüências que a doença pode acarretar (dor, morte, gasto material, interrupção de atividades, perturbação nas relações familiares e sociais, e outras)" ${ }^{(15)}$.

Embora a QV tenha enfoques bastante amplos, quais sejam biológicos, psicológicos, emocionais, sociais, econômicos e até ecológicos, neste estudo ela é avaliada com a aplicação do SF36® (The Medical Outcomes Study 36-item Short-Form Health Survey), um instrumento genérico de avaliação de saúde traduzido e validado no Brasil ${ }^{(16)}$

A percepção da gravidade da doença manifestada pelo paciente, assim como a qualidade de vida na visão deste doente crônico, são consideradas neste trabalho, da maior importância para nortear a avaliação e intervenção do enfermeiro.

\section{OBJETIVOS}

Os objetivos deste estudo foram:

- Verificar a interferência da doença crônica sobre a QV de hipertensos adultos hospitalizados, utilizando instrumento genérico de avaliação. - Confrontar os dados relacionados a QV com a percepção de gravidade da doença, manifestada pelos pacientes estudados.

\section{CASUÍSTICA E MÉTODO}

Trata-se de um estudo descritivo, transversal. 
População e local do estudo: pacientes de ambos os sexos, com idade superior a 18 anos, em tratamento de HA, internados no setor de Clínica Médica do Núcleo de Hospital Universitário (NHU) da Universidade Federal de Mato Grosso do Sul (UFMS), em Campo Grande - Mato Grosso do Sul.

Amostra: constituiu-se de 83 pacientes, sendo 38 homens e 45 mulheres.

Critérios de inclusão: estar em tratamento de HA como doença de base ou associada à diabete mellitus, coronariopatia, nefropatia (esta última secundária à $\mathrm{HA}$ ); estar orientado no tempo e no espaço; ser capaz de se comunicar verbalmente; ter idade acima de 18 anos; consentir em participar da pesquisa.

Instrumento de Coleta de Dados: constituiu-se de formulário submetido a pré-teste, quando aplicado a 08 pacientes com as mesmas características da população do estudo, visando identificar possíveis falhas na formulação das questões, buscar clareza e verificar a necessidade de adequação do vocabulário.

No instrumento, destacam-se 03 partes:

Parte I: dados sócio-demográficos.

Parte II: dados sobre Qualidade de Vida: foi utilizado o SF36® (The Medical Outcomes Study 36-item Short Form Health Survey), traduzido e validado no Brasil ${ }^{(16)}$. A pesquisadora que 0 traduziu e validou refere-se ao instrumento como bem desenhado, multidimensional, útil para este tipo de avaliação, de fácil administração e compreensão, bem como de sua utilização por outros investigadores em diversas patologias crônicas.

0 SF36® é formado por 36 itens que englobam nove escalas: Capacidade Funcional (CF) com 10 itens; Desempenho Físico (DF) com 04 itens; Dor (DR) com 02 itens; Estado Geral de Saúde (GS) com 05 itens; Vitalidade (VT) com 04 itens; Aspectos Sociais (AS) com 02 itens; Aspectos Emocionais (AE) com 03 itens; Saúde Mental (SM) com 05 itens; Evolução do Estado de Saúde (EV), referese a avaliação comparativa entre as condições de saúde atual e a de um ano atrás, com 01 item.

Parte III: dados de percepção de gravidade da doença.

Coleta de Dados: após a emissão de autorização pelo Conselho Diretivo do NHU/UFMS e parecer favorável dos competentes Comitês de Ética em Pesquisa da UFMS e UNIFESP-EPM, a coleta de dados foi realizada.

Período: de 18 de setembro de 1998 a 18 de janeiro de 1999.

Os dados foram coletados pelo método de auto-relato por meio de entrevista com preenchimento de formulário com questões estruturadas e semi-estruturadas ${ }^{(17)}$. A opção pelo formulário deveuse ao baixo nível de escolaridade observado previamente na população a ser estudada.

Consentimento informado: por meio da Carta de Informação todos os participantes foram esclarecidos sobre o estudo e seus objetivos, direito de não participação, garantia de que sua assistência não seria afetada caso ele não aceitasse participar, nem pelas respostas fornecidas, caso aceitasse. Foi assegurado anonimato e sigilo. Os que aceitaram participar assinaram o Termo de Consentimento Pós-Informação.

Procedimentos para computação e tabulação dos dados: os dados sócio-demográficos e de percepção de gravidade da doença foram codificados e devidamente agrupados em freqüência simples e porcentagem.

0 tratamento dos dados de QV seguiu as orientações contidas no The SF-36尺 - Health Survey Manual ${ }^{(18)}$. Inicialmente foi estabelecida a correspondência de cada item ou questão à sua respectiva escala. Numa outra etapa, foi realizada a recodificação de 10 itens, os quais no instrumento da pesquisa, apareceram invertidos na pontuação ou desiguais em número de opções de uma mesma escala. Exemplo: escala "DOR", composta pelos itens 7 e 8 . No item 7, com seis alternativas de resposta, numeradas de 01 a 6 e no item 8 , com 5 alternativas numeradas de 1 a 5 . Foi realizada a uniformização para 6 alternativas em ambas as questões. Visando alcançar uma adequação linear, isto é, um alinhamento de valores em todas as questões, a recodificação foi realizada atribuindo-se um escore para cada alternativa, em que o escore de maior valor correspondeu a um melhor estado de saúde e o menor ao pior estado de saúde.

Os dados de QV foram então computados, considerando os valores recodificados, fazendo relação com os dados colhidos na entrevista. Os escores dos itens de cada escala foram somados, os quais correspondiam às respostas dos entrevistados. Por exemplo, considerando a escala GS, foram somados os escores referentes as respostas dos 83 pacientes aos itens correspondentes à escala GS, ou seja item 1, Ila, Ilb, Ilc e lld (para cada item o paciente atribuiu uma resposta), perfazendo no total 415 respostas. $\mathrm{Na}$ seqüência, tendo os " $n$ " determinados, os respectivos percentuais foram calculados.

$\mathrm{Na}$ etapa seguinte, procedeu-se a transformação de cada escore numa escala de 0 a 100. Esta transformação compreendeu a conversão do valor mínimo e valor máximo possível para cada item (padronizados segundo o Manual para cálculo das escalas transformadas), em cada escala e para cada paciente. Escores entre estes valores representaram a porcentagem possível de ser alcançada.

Para o cálculo dos escores transformados, os passos foram:

1. Calcular cada uma das escalas (CF, DF, GS, DR, VT, AS, AE, SM): soma dos pontos obtidos de cada item relativo à correspondente escala, para cada paciente. 
2. Utilizando-se os valores mínimos e máximos possíveis, para cada item calcular o valor transformado, aplicando-se a fórmula:

$$
Y_{i j}=\frac{X_{i j}-\min j}{\text { Máxj- minj }} \times 100
$$

Onde:

I= 1,2,3,...83 (Índice do paciente).

$\mathrm{J}=\mathrm{CF}, \mathrm{DF}, \mathrm{GS}$... (cada uma das escalas)

Yij= valor transformado do paciente i, escala $\mathrm{j}$.

$X i j=$ valor da escala $\mathrm{j}$, do paciente $\mathrm{i}$.

Min $\mathrm{j}=$ valor mínimo possível para a escala $\mathrm{j}$.

Max j = valor máximo possível para a escala j.

Tratamento e análise estatística dos dados: os dados computados foram submetidos a tratamento estatístico descritivo. Para as variáveis classificatórias foram aplicados os testes de Razão de Verossimilhança e Qui-quadrado. Para análise dos dados de QV foram calculadas Média e Desvio Padrão e aplicados testes de Análise de Variância, Teste de Tuckey e Teste de Kruskal-Wallis. Optou-se por discutir os dados de QV pelo estudo estatístico, ao invés de fazê-lo pelas escalas, devido ao número diferente de escores existente para cada uma delas. 0 estudo estatístico, correspondeu a comparação entre a percepção de gravidade da doença com cada uma das oito (8) escalas: GS, CF, DF, AE, AS, DR,VT e SM. Quanto a escala EV, conforme instrução contida no Health Survey SF36® Manual, a discussão foi realizada pelos percentuais. 0 nível de significância adotado foi de 0,05, sendo considerados estatisticamente significantes os resultados dos cruzamentos de dados relativos a percepção do paciente quanto à gravidade da doença com os demais dados, cujos valores de p foram inferiores a 0,05 .

\section{RESULTADOS E DISCUSSÃO}

Os pacientes ( $\mathrm{N}=83$ ) em sua maioria eram do sexo feminino (54,2\%); os homens representaram $45,8 \%$ da amostra. A maioria dos pacientes, encontrava-se na faixa etária entre 50 e 69 anos, perfazendo um percentual de 57,8\%.Quando estabelecida a comparação da percepção de gravidade da doença, com a idade e com o sexo não houve diferença estatisticamente significante $(p>0,05)$. Segundo a OMS , a pressão arterial se eleva na medida em que são acrescidos anos de vida à populaçãa ${ }^{(19)}$. Considerando que no Brasil os dados demográficos apontam para um envelhecimento populacional que avança rapidamente, pode-se vislumbrar uma tendência para o futuro de um número elevado de hipertensos. A HA apresenta-se com maior prevalência no sexo masculino até 45 a 50 anos, sendo que a partir desta idade a prevalência é maior no sexo feminino devido aos fatores hormonais da menopausa $(19,20)$.

A maioria tinha um companheiro $(63,9 \% n=53)$. Destes, $57,8 \%$ moravam na companhia de seus cônjuges; os demais e os muitos dos que não tinham cônjuge viviam com os filhos e/ou mãe $(29,0 \%)$ e com outras pessoas ou sozinhos $(13,2 \%)$. Ao realizar a comparação entre a percepção de gravidade da doença e situação conjugal e familiar, não houve diferença estatisticamente significante $(p>0,05)$. Porém, observou-se que os pacientes que moravam na companhia de alguém, seja de companheiro, pais, filhos, parentes ou amigos, tinham maior percepção da doença como grave (em média, 57,6\%); a maioria dos que moravam sozinhos percebiam a HA como doença sem gravidade $(60,0 \%)$.

Um percentual significativo de pacientes não possuía dependentes $(n=36 ; 44,4 \%)$. Os demais possuíam 01 dependente (24,7\%), 02 dependentes (11,1\%), 03 dependentes (16,1\%) e mais de 03 dependentes num percentual menor $(3,7 \%)$. Isto deveuse, provavelmente, ao número de pacientes idosos no grupo estudado. Analisando a associação entre a percepção de gravidade da doença e o número de dependentes, percebeu-se que a maioria dos que não acreditavam na gravidade da doença, não possuía dependentes $(69,2 \%)$. Os pacientes que acreditavam na gravidade da doença, possuíam dependentes. A diferença foi estatisticamente significante quando comparada a referida percepção em relação ao número de dependentes $(p<0,05)$. 0 presente estudo demonstra que o elo afetivo com os filhos modificou a percepção do paciente em relação à sua patologia. Devido a escassa literatura relacionando a HA com as questões afetivas do ser humano, este é um aspecto que merece a iniciativa de novas pesquisas e aprofundamento a respeito do assunto.

No grupo estudado, a cor parda foi a cor de pele mais freqüente ( $n=40,48,2 \%)$, seguida pela cor branca $(41,0 \%)$ e negra $(10,8 \%)$. Este resultado deveu-se, provavelmente, às características regionais da população, pela forte miscigenação racial advinda do cruzamento entre brancos, negros e índios. Na literatura explorada vários autores indicam o predomínio da HA entre negros, justificada em fatores genéticos e hereditários ${ }^{(19)}$. Os estudos sobre prevalência em negros se concentram na sociedade americana, sendo que 0 Brasil carece de estudos neste aspecto, pois a composição racial dos brasileiros é muito heterogênea ${ }^{(20)}$. 0 estudo estatístico não revelou significância quando comparada esta variável com a percepção de gravidade da doença ( $p>0,05)$.

Dos pacientes estudados, 69,9\% $(n=58)$ procedia de Campo Grande e $26,5 \%$ do interior do Estado de Mato Grosso do Sul. 0 fato do Hospital Universitário da UFMS ser um hospital de referência local e regional, em convênio com o Sistema Único de Saúde e o único hospital público da região que atende emergências, provavelmente é a razão para os números encontrados. 
Estatisticamente não houve diferença significante para esta variável $(p>0,05)$.

Os resultados demonstraram grau de escolaridade muito baixo. A maioria não tinha completado o I Grau ( $n=77 ; 92,8 \%$ ) e os demais tinham concluído, no máximo, a primeira série do II Grau. Ao proceder-se a comparação entre a percepção de gravidade da doença e os graus de escolaridade, não houve significância, mas observou-se que os pacientes com maior nível de escolaridade, eram também os mais esclarecidos quanto à gravidade da $\mathrm{HA}$, pois todos a percebiam como grave e incurável. A predominância da baixa escolaridade encontrada neste estudo assume relevância quando se considera a abordagem a estes pacientes, que deve ser em linguagem simples e compatível com o grau de entendimento. Igualmente importante, quando se confirma que pacientes com maior grau de escolaridade, apresentam uma percepção mais esclarecida da doença.

Constatou-se que o nível sócioeconômico do grupo pesquisado era baixo, representado pela renda familiar de no máximo 02 salários mínimos para $57,3 \%$ dos pacientes $(n=47)$. $\mathrm{Na}$ comparação da percepção de gravidade da doença com a renda familiar não houve diferença estatisticamente significante $(p>0,05)$.

Quanto ao estudo estatístico realizado, quando comparados os resultados de QV (por escalas) à percepção que 0 paciente tem da sua doença, houve diferença significante apenas para as escalas Estado Geral de Saúde (GS) e Capacidade Funcional (CF) $(p<0,05)$. Avaliando a comparação realizada, observou-se que nestas escalas em que houve significância estatística (tanto na escala GS como na (F), a percepção da HA como doença sem gravidade teve distribuição distinta dos demais grupos. Isto é, a média correspondente a estas escalas é maior entre os que acreditam que a doença não é grave.

No entanto, quanto a evolução do estado de saúde (escala EV), comparando a um ano atrás, 26,5\% referiram estar melhores hoje. Mais da metade (50,6\%), referiu que hoje está pior e 14,5\% informaram que estão muito piores. Apenas $8,4 \%$ acreditam que estão no mesmo estado de saúde quando feita a esta comparação. Portanto, isoladamente, este resultado significaria piora da QV referida.

Sendo assim, cabe considerar que qualidade de vida, coletiva ou individual, é relativa a sentimentos do homem sobre sua própria saúde ou sobre outros aspectos de sua vida ${ }^{(21-22)}$; e que para avaliar a qualidade de vida de alguém, considera-se que a mesma pode ser influenciada pela percepção que este indivíduo tem do seu estado de saúde, pois sua satisfação é influenciada pela bagagem de crenças e valores ${ }^{(14)}$.

Considerando que estamos tratando de pacientes hipertensos, e portanto com limitações impostas pela condição de cronicidade da doença, que geralmente leva a mudanças permanentes ${ }^{(23)}$, muitas vezes de difícil aceitação, e que produzem sentimentos de tristeza, raiva, agressividade, hostilidade ${ }^{(24)}$, conhecer os resultados correspondentes aos diversos aspectos de QV estudados nesta pesquisa, é de suma importância ao profissional de saúde, uma vez que deseja-se ao hipertenso sob seus cuidados, que o mesmo venha a conhecer sua doença, desenvolva a autoresponsabilidade, assuma seu papel ativo, modifique seus comportamentos em relação à saúde, ajuste-se às limitações físicas e aos relacionamentos sociais e mantenha esperança e alegria de viver.

Convém destacar que os dados de QV neste estudo, referem-se à percepção do paciente, como ele interpreta sua condição de hipertenso em termos de QV e de gravidade da doença. A severidade dos sintomas e intercorrências clínicas podem alterar a QV do portador de doença crônica ${ }^{(14)}$.

Desse modo, um estudo ${ }^{(25)}$ relatando qualidade de vida sugere que os resultados sejam analisados no contexto da população investigada. Assim sendo, vale lembrar que todos os pacientes estudados encontravam-se hospitalizados, portanto recebendo 0 mesmo nível de atenção à saúde. Por outro lado, além da doença de base, havia a presença de complicações, descontrole metabólico, tempo de evolução da doença e outras morbidades associadas, que também causavam sintomas, desconfortos e limitações aos sujeitos deste estudo.

Outro estudo exploratório desenvolvido em um hospital geral de ensino, com o objetivo de identificar mudanças ocorridas no estilo de vida de 45 pacientes em condições crônicas de saúde, demonstrou que o indivíduo se depara com "novas incumbências", e problemas de ordem social, econômica e pessoal, muitas vezes de difícil enfrentamento. Demonstrou também que as alterações no estilo de vida do paciente pode ser gradual ou cumulativa, mas não ocorrem todas ao mesmo tempo, permitindo portanto, que a enfermagem adote uma estratégia específica de assistência que ajude-o a se adaptar as necessidades de mudança e manter controle de sua situação( $0^{(23)}$.

Uma pesquisa realizada com 300 hipertensos, identificou crenças e hábitos de vida que implicam em agravos ao paciente de ordem física, familiar e social: alterações de sono (22,9\%), na alimentação $(21,1 \%)$, nas atividades físicas $(21,1 \%)$, no relacionamento familiar $(13,6 \%)$ e no trabalho (19,3\%). Identificou também que $68,0 \%$ dos pacientes acreditavam na gravidade da doença, 31,4\% não sabiam responder ao questionamento e apenas $0,6 \%$ não acreditavam na gravidade da doença. Segundo os autores, "estes dados, por apresentarem estreita correlação entre si, poderiam ocasionar desajustes na vida destas pessoas, com conseqüente diminuição das atividades domésticas ou ligadas ao 
trabalho e influenciar negativamente sobre a aderência ao tratamento" ${ }^{(9)}$.

No presente estudo, a percepção "sem gravidade" em destaque para as escalas GS e CF, pode significar que a QV é boa, sob a ótica do paciente. Mediante este resultado, pode-se inferir que a percepção "sem gravidade" também pode influenciar na adesão ao tratamento. Isto leva a considerar-se que, frente aos resultados anteriormente descritos, o paciente que percebe a hipertensão como doença sem gravidade e boa QV, provavelmente é o que mais necessita de assistência. Certamente ele não valoriza a doença porque a mesma não apresenta alterações visíveis. Talvez em outras doenças, a percepção de QV boa representaria menor preocupação em termos de abordagem à saúde.

Além da percepção, inúmeros outros aspectos podem influenciar a aderência ao tratamento ${ }^{(24,26)}$. Mas se tal possibilidade existe, então a avaliação da percepção do paciente em relação à enfermidade deve ser interpretada pelo enfermeiro, para que a intervenção seja apropriada. A avaliação da QV amplia as decisões clínicas do enfermeiro, as quais se estendem aos programas $\mathrm{e}$ políticas assistenciais ${ }^{(7)}$. Outros autores também são unânimes em afirmar que o fracasso de muitos programas, deve-se ao fato de que os mesmos estão ancorados na percepção dos profissionais de saúde, no método autoritário de prestar assistência, com intervenções desconectadas das condições sociais de qualidade de vida, e desta forma, havendo pouca investigação e valorização das reais necessidades do cliente, suas crenças e sua motivação( ${ }^{(27-29)}$.

\section{CONCLUSÃO}

Os resultados desta investigação permitiram concluir que: - Em concordância com a bibliografia, o fator idade concorre para o

\section{REFERÊNCIAS BIBLIOGRÁFICAS}

1. Lessa I. Doenças não-transmissíveis. In. Rouquayrol MZ, organizadora. Epidemiologia e saúde. Rio de Janeiro (RJ): MEDSI; 1994. p. 269-79.

2. Colombo RCR, Aguillar OM. Estilo de vida e fatores de risco de pacientes com primeiro episódio de infarto agudo do miocárdio. Rev Latino-am Enfermagem 1997 abril; 5(2):69-82.

3. Ribeiro AB. Atualização em hipertensão arterial - clínica, diagnóstico e terapêutica. São Paulo (SP): Atheneu; 1996.

4. Pessuto J, Carvalho EC. Fatores de risco em indivíduos com hipertensão arterial. Rev Latino-am Enfermagem 1998 janeiro; 6(1):33-9. aumento da pressão arterial, isto é, a PA se eleva na medida em que anos de vida são acrescidos.

- 0 elo afetivo com os filhos modifica a percepção da doença, pois os que tinham dependentes acreditavam que a HA é grave.

- Há necessidade de linguagem simples e de fácil entendimento na abordagem a esta clientela, devido ao grau de escolaridade baixo $(92,8 \%)$.

- Devido a renda familiar diminuta (no máximo 2 SM para 57,3\%), e portanto, dificuldade em adquirir medicação, esta deve ser favorecida. As orientações relativas ao estilo de vida devem ser adaptadas a condição socioeconômica dos pacientes.

- Embora a hipertensão seja doença grave que leva a complicação de órgãos nobres como cérebro e rins, o estudo estatístico revelou que, na visão do hipertenso, sua doença não é grave e sua QV é boa. Estas percepções podem interferir na aderência ao tratamento. Provavelmente, o paciente com esta percepção é o que mais necessita de assistência de enfermagem.

- A avaliação e interpretação das percepções do paciente são instrumentos que o enfermeiro deve lançar mão para direcionar a sua intervenção. Acredita-se que alcançando maior compreensão, sobre a problemática que permeia a condição de ser portador de uma doença crônica, pode-se definir estratégias mais apropriadas de assistência ao hipertenso.

- A promoção da saúde, por meio de ações educativas, pode gerar auto-responsabilidade, adoção de um estilo de vida saudável, redução dos fatores de risco, redução dos sintomas da doença crônica e melhoria da QV.

- Resultados sobre severidade da doença e qualidade de vida percebidas poderão ser diferentes após a implementação de uma assistência bem orientada e especificamente direcionada a este tipo de paciente.

5. Yusuf HR, Giles WH, Croft JB, Anda RF, Casper ML. Impact of multiple risk factor profiles on determining cardiovascular disease risk. Preventive Medicine 1998; 27:1-9.

6. Sousa ALL. Prevalência da Hipertensão Arterial referida, percepção de sua origem e formas de controle em área metropolitana de São Paulo - SP (1989-1990). [dissertação]. São Paulo (SP): Faculdade de Saúde Pública/USP; 1999.

7. Sociedade Brasileira de Hipertensão. Consenso Brasileiro de Hipertensão Arterial (III). HiperAtivo 1999; 6(1):67-106.

8. Santos AS. Papel da educação em saúde no diabetes, na hipertensão arterial e na terceira idade. Rev 0 mundo da saúde 1998 janeiro/fevereiro; 22(22):11-7.

9. Car MR, Pierin AMG, Mion Junior D, Giorgi DMA. Crenças de saúde do paciente com hipertensão arterial. Rev Paul Enfermagem 1998 abril/maio/junho; 8(2):4-7. 
10. Ministério da Saúde (BR).Controle da Hipertensão Arterial: uma proposta de integração ensino-serviço. Rio de Janeiro (RJ): CDCV/ NUTES; 1993.

11. Borestein MS, organizadora. Manual de hipertensão. Porto Alegre (RS): Sagra Luzzatto; 1999.

12. Nicholls MG, Richards AM, Begg EJ. Hipertension and heart failure. Exp. Med. Adv Biol 1997; 432: 273-9.

13. Rivera AJB, Roldán FLT, Casanova LIS, Beltran MM. Efecto de una intervencion educativa en la calidad de vida del paciente hipertenso. Salud Pública de México 1998 noviembre/deciembre; 40(6):503-9.

14. Trentini M, Silva DGV, Martins CR, Antônio MC, Tomaz CE, Duarte R. Qualidade de vida dos indivíduos com doenças cardiovasculares crônicas e diabetes mellitus. Rev Gaúch Enfermagem 1990 julho;11(2):20-7.

15. Della Coleta MF. 0 modelo de crenças em saúde: uma aplicação a comportamentos de prevenção e controle da doença cardiovascular. [dissertação]. Brasília (DF): Universidade de Brasília; 1995.

16. Ciconelli RM. Tradução para o português e validação do questionário genérico de avaliação de qualidade de vida "Medical Outcomes Study 36-item Short-form health survey (SF-36). [dissertação]. São Paulo (SP): Universidade Federal de São PauloEscola Paulista de Medicina; 1999.

17. Polit DF, Hungler BP. Fundamentos de pesquisa em enfermagem. $3^{\mathrm{a}}$ ed: Porto Alegre (RS): Artes Médicas; 1995.

18. Ware JE Junior. SF-36® health survey manual. Boston: Massachusets; 1993.

19. Organization World of Health. Control de la Hypertension. Informe de um Comitte de Expertos de la OMS. Ginebra, Switzerland: OMS; 1996.
20. Lessa I, Hage EC, Mendonça GAES, Franco U, Teixeira MTB, Barreto ML et al. 0 adulto brasileiro e as doenças da modernidade - epidemiologia das doenças crônicas não-transmissíveis. São Paulo (SP): HUCITEC/ABRASCO; 1998.

21. Forattini OP. Ecologia, epidemiologia, sociedade. São Paulo (SP): Artes Médicas; 1992.

22. Gill TM, Feinstein AR. A critical appraisal of the quality of qualityof-life measurements. JAMA 1994 August; 272(8):619-26.

23. Trentini M, Silva DGV da, Leimann AH. Mudanças no estilo de vida enfrentadas por pacientes em condições crônicas de saúde. Rev Gaúch Enfermagem 1990 janeiro; 11(1):18-28.

24. Pierin AMG, Car MR. Instrumento de consulta de enfermagem a pessoas com hipertensão arterial em tratamento ambulatorial. Rev Esc Enfermagem USP 1992 março; 26(1):17-32.

25. Peña MCG, Morales HR, Espinosa JG, Gutiérrez AF, García CM. La calidad de vida en el paciente diabético tipo Il y factores relacionados. Rev Médica del Instituto Mexicano del Seguro Social 1995 enero; 33(3): 293-8.

26. Car MR, Pierin AMG, Aquino VLA. Estudo sobre a influência do processo educativo no controle da hipertensão arterial. Rev Esc Enfermagem USP 1991 dezembro; 25(3):259-69.

27. Zanetti ML, Mendes IAC. Tendência do locus de controle de pessoas diabéticas. Rev Escola de Enfermagem USP 1993 janeiro; 27(2):246-62.

28. Souza MMF de, Kalichman AO. Vigilânica à saúde: epidemiologia, serviços e qualidade de vida. In: Rouquayrol MZ, organizadora. Rio de Janeiro (RJ): Medsi; 1994. p. 467-76.

29. Trentini M, Dias LPM. Ser mais - uma possibilidade de promover saúde na prática educativa. Acta Paul Enfermagem 1997 janeiro/ abril; 10(1):53-61. 